\title{
Gastric Tuberculosis
}

\author{
Naresh Kumar Seetlani1, Khalid Imran1, Qurat ul Ain Hafeez1, Asif Ali1, Pyar Ali Fazil1, \\ Bareeha Shaukat Sultan² and Sulhera Khan²
}

\begin{abstract}
Gastric tuberculosis is a very rare disease posing a diagnostic challenge to physicians, pathologists and radiologists. It usually occurs in females aged 25 to 45 years. Symptoms include epigastric pain, vomiting, fever, weight loss, upper gastrointestinal (GI) bleed and gastric outlet obstruction. High index of suspicion and endoscopic or endoscopic ultrasound guided biopsies may help in making an early diagnosis, particularly in patients with non-healing gastric ulcers. We report a case of gastric tuberculosis in a 55-year male, who presented with persistent vomiting, epigastric pain and significant weight loss. His upper GI endoscopy finding with histopathology results favoured the diagnosis of gastric tuberculosis. He was successfully treated with antituberculous regimen.
\end{abstract}

Key Words: Granuloma, Persistent vomiting, Stomach, Tuberculosis.

\section{INTRODUCTION}

According to the World Health Organization Global Tuberculosis Report (2018), about 10.0 million people were infected with tuberculosis (TB) in 2017 and about 55\% of these cases were from India, Indonesia, China, Philippines and Pakistan. Pakistan ranks 5th among the highest burdened countries of tuberculosis worldwide with an annual incidence of 525,000 new TB cases. Tuberculous involvement of the gastrointestinal system accounts for around $12 \%$ of extra-pulmonary tuberculosis and is usually seen in immunocompromised patients. $1^{1,2}$ It usually presents in one of the four ways as visceral tuberculosis which involves solid organs; gastrointestinal tuberculosis; peritoneal tuberculosis; and tuberculous lymphadenopathy. 2,3 The commonest sites include the ileocaecal region $(65 \%)$ followed by jejunum and colon $(10.8 \%)$, but involvement of esophagus $(0.2-1 \%)$, stomach $(0.4-2 \%)$ and duodenum $(2-2.5 \%)$ is rare. ${ }^{3}$ The diagnosis of these rare cases of tuberculosis may be difficult without specific radiological, endoscopic and clinical features. Here, we report a case of gastric tuberculosis.

\section{CASE REPORT}

A 55-year male resident of Karachi presented with persistent episodes of non-bile stained vomiting and epigastric pain for three months, associated with

\footnotetext{
1 Department of Medicine, Dow Medical College, Dr. Ruth K.M. Pfau, Civil Hospital, Karachi, Pakistan

2 Medical Student, Dow Medical College, Dr. Ruth K.M. Pfau, Civil Hospital, Karachi, Pakistan

Correspondence: Dr. Naresh Kumar Seetlani, Department of Medicine, Dow Medical College, Dr. Ruth K.M. Pfau,

Civil Hospital, Karachi, Pakistan

E-mail: drnaresh2004@yahoo.com

Received: June 11, 2018; Accepted: February 08, 2019
}

significant weight loss. He had no history of fever or respiratory symptoms. He was on antiemetics and antacids. There was no family history of tuberculosis or malignancy. On examination, patient was dehydrated but there were no anemia, jaundice, cervical lymphadenopathy or hepatosplenomegaly. His hemato-logical findings were unremarkable; erythrocyte sedimentation rate was raised, i.e. $30 \mathrm{~mm}$ after first hour. Serology for hepatitis $B$, hepatitis $C$ and HIV were negative. His serum albumin level was within the normal limits and no abnormal finding was seen on the chest X-ray.

Patient was prepared for upper gastrointestinal endoscopy, which showed a large ulcerated area with raised margins occupying most of lesser and greater curvature extending towards the pylorus and occluding it partially (Figure 1). Multiple biopsies were taken for histopathology. The results revealed multiple fragments of gastric mucosa with crushing artifacts, moderate to severe active gastritis with crypt abscesses. Multiple foci of ulcer slough and ill-defined epitheloid cell granulomas with multinucleated giant cells were also seen in the mucosa. The lamina propria exhibited moderate infiltration of mononuclear cells having moderate activity (Figure 2). No convincing evidence of metaplasia, dysplasia or malignancy was noted. A computed tomography scan of whole abdomen with contrast was done which showed thick walled stomach with hetreogenous enhancement and multiple solid enhancing lymph nodes at celiac axis, perigastric region, along lesser curvature, pre-pyloric and peripancreatic regions, largest measuring $1 \mathrm{~cm}$ in prepyloric region. Minimal non-tapable ascites was also identified. The small and large bowel, rectum and anus appeared normal. Patient was put on anti-tuberculosis regimen containing four drugs (rifampicin, isoniazid, ethambutol and pyrazinamide) with vitamin B6 therapy and was closely followed for compliance and response to treatment. He improved with the treatment; and vomiting decreased with passage of time. 


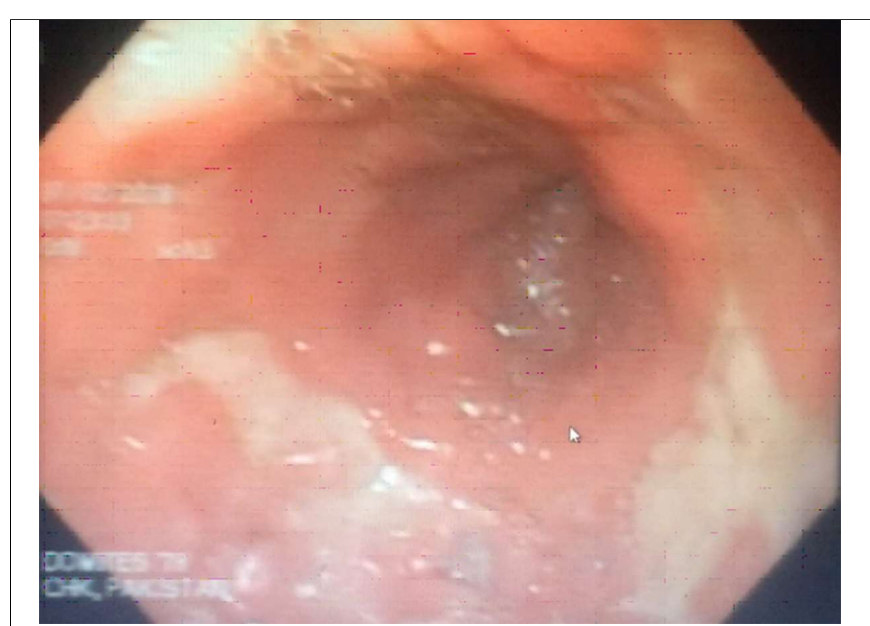

Figure 1: Endoscopic picture showing large ulcers with raised margins occupying most of the lesser and greater curvatures.

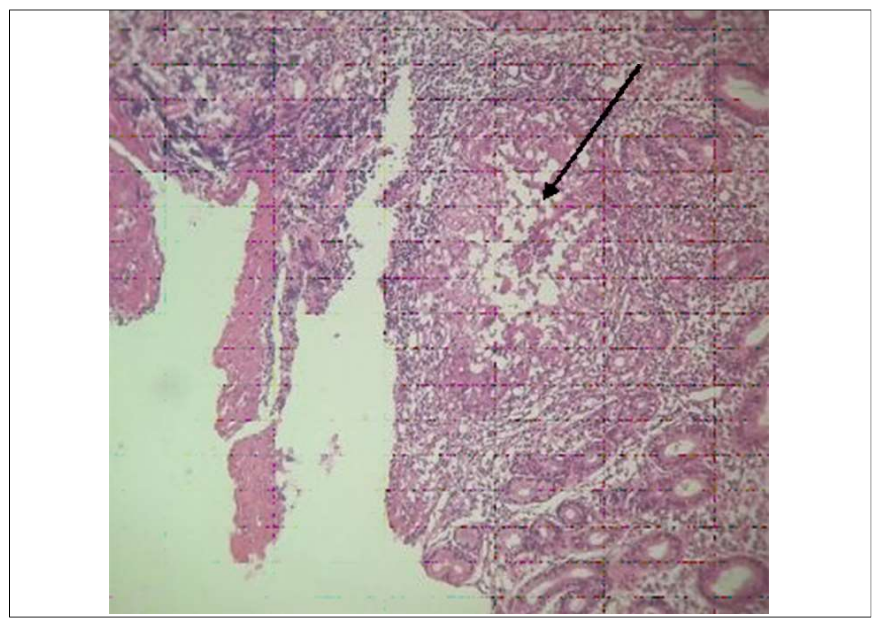

Figure 2: Histopathological slide showing epitheloid cell granulomas and multinucleated giant cells.

\section{DISCUSSION}

This case has several uncommon features including the rare site in an uncommon age and gender. According to literature, abdominal TB is predominantly seen in females $25-45$ years of age, 1 but this patient was a male aged 55 years, which is not common.

In abdominal tuberculosis, involvement of stomach is very rare. Debi et al. explained the reasons of its rarity as bactericidal nature of gastric acid, scarcity of lymphoid tissue in gastric wall, and thick intact gastric mucosa of stomach. ${ }^{3}$

Malikowski et al. explained the pathogenesis of abdominal tuberculosis as invasion of pathogenic bacteria and then granulomatous inflammation, which lead to ulceration, bleeding and perforation. ${ }^{2}$ In this case, large ulcers were formed by granulomatous inflammation. The most common sites of upper GI TB are lesser curvature of antrum and prepyloric region, while duodenal involvement is rare. 3,4 In this patient, pylorus was involved with lesser and greater curvature.
Clinically, gastric ulcer caused by TB resembles cancer or peptic ulcer disease which may be misleading. A report by Sandhya et al. has shown a case of isolated gastric TB, which was being treated with a diagnosis of chronic peptic ulcer and presenting with its complication, i.e. gastric outlet obstruction. ${ }^{4}$

The symptoms of gastric tuberculosis differ, depending on the site involved. ${ }^{5}$ Patients may present with epigastric pain, vomiting, fever, weight loss, upper Gl bleed and gastric outlet obstruction. ${ }^{3}$ This patient presented with same features except gastric outlet obstruction feature and bleeding. Arabi et al. presented a case from Sudan with projectile vomiting and epigastric pain with weight loss, which on histopathology, diagnosis of gastric TB was confirmed. 1

Lymphoma, carcinoma and other infections like syphilis etc. can be the differential diagnosis of gastric tuberculosis. ${ }^{3}$

Eray et al. reported from Turkey a case of gastric TB mimicking gastric cancer and they found the poor yield of endoscopic biopsy for the confirmation of diagnosis; and explained that it rarely reveals granulomas because of sub-mucosal site of the ulcerated lesion. 5 Sharma et al. from India diagnosed a rare case of gastric tuberculosis which was mimicking a sub-mucosal tumor after surgery and they found that endoscopic ultrasonography as an excellent tool for classifying the sub-mucosal lesion and also for sampling for the cytological confirmation of diagnosis. 6 Malikowski et al. from United States made a fluid and tissue analysis as the definitive criteria for diagnosis. 2 Upadhyaya et al. studied five patients of gastro duodenal TB with gastric outlet obstruction; they found that the most frequent presenting complaints were vomiting, failure to thrive and weight loss. Fine needle aspiration cytology (FNAC) were done on all patients, which showed granulomas in four cases. ${ }^{7}$ Another study found endoscopic balloon dilatation along with antituberculous treatment is effective in patients with gastric outlet obstruction having granulomatous inflammation with or without demons-tration of AFB. 8 In one of the study from Uttarkhand, 68 patients of abdominal TB were reviewed and found that the commonest cause was intestinal TB (45.59\%), but not a single esophageal, gastric or duodenal TB case was seen. ${ }^{9} \mathrm{~A}$ case control study found that long-term use of $\mathrm{H} 2$ blockers and PPIs have association with TB infection. 10

With antituberculous therapy, surgery needs to be done only in complicated cases i.e. gastric outlet obstruction, hematemesis, perforation or gastro-bronchial fistula. This patient was successfully managed without any surgical intervention.

\section{REFERENCES}

1. Arabi NA, Musaad AM, Ahmed EE, Ibnouf MM, Abdelaziz MS. Primary gastric tuberculosis presenting as gastric outlet 
obstruction: A case report and review of the literature. $J$ Med Case Rep 2015; 9:265.

2. Malikowski T, Mahmood M, Smyrk T, Raffals L, Nehra V. Tuberculosis of the gastrointestinal tract and associated viscera. J Clin Tuberc Other Mycobact Dis 2018; 12:1-8.

3. Debi U, Ravisankar V, Prasad KK, Sinha SK, Sharma AK. Abdominal tuberculosis of the gastrointestinal tract: Revisited. World J Gastroenterol 2014; 20:14831-40.

4. Sandhya V, Balaji BD, Pradeep US, Alok SC. Isolated gastric tuberculosis masquerading as chronic peptic ulcer: A case report. Int J Med Res Health Sci 2015; 4:896-8.

5. Eray IC, Rencüzocullari A, Yalav O, Dalci K, Kakil E, Bagir E, et al. Primary gastric tuberculosis mimicking gastric cancer. Turk J Surg Ulus Cerrahi Derg 2015; 31:177-9.

6. Sharma V, Rana S, Gunjan D, Chhabra P, Sharma R, Bhasin D.
Primary gastric tuberculosis mimicking a submucosal tumor. $J$ Digest Endos 2015; 6:130-2.

7. Upadhyaya VD, Kumar B, Lal R, Sharma MS, Singh $M$, Rudramani. Primary duodenal tuberculosis presenting as gastric-outlet obstruction: Its diagnosis. Afr J Paediatr Surg 2013; 10:83-6.

8. Puri AS, Sachdeva S, Mittal VV, Gupta N, Banka A, Sakhuja P, et al. Endoscopic diagnosis, management and outcome of gastroduodenal tuberculosis. Indian J Gastroenterol 2012; 31: 125-9.

9. Hatwal D, Kumar A, Batra N. Abdominal tuberculosis in Uttarakhand: A diagnostic dilemma. Indian J Pathol Oncol 2017; 4:188-92.

10. Hsu WH, Kuo CH, Wang SS, Lu CY, Liu CJ, Chuah SK, et al. Acid suppressive agents and risk of mycobacterium tuberculosis: Case-control study. BMC Gastroenterol 2014; 14:91. 